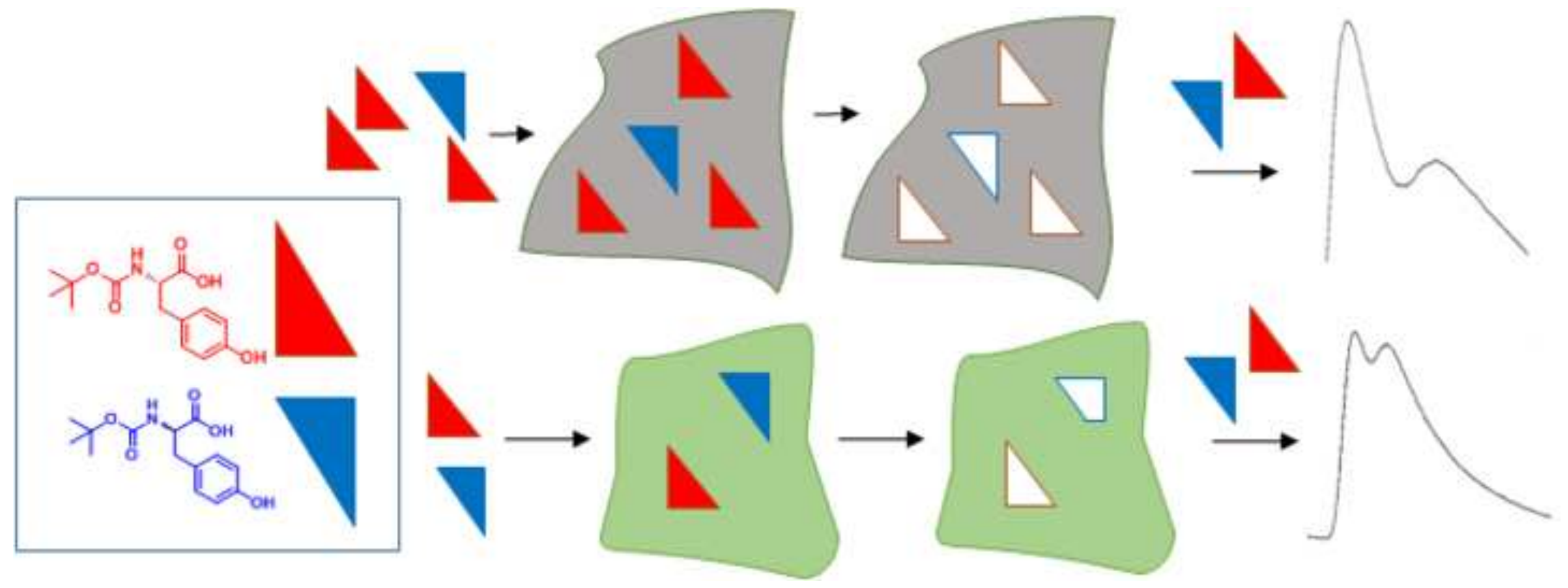




\title{
Scalemic and Racemic Imprinting with a Chiral Crosslinker
}

\author{
Britney Hebert, Danielle S. Meador, David A. Spivak* \\ Department of Chemistry, Louisiana State University, Baton Rouge, LA 70803
}

\begin{abstract}
The development of molecularly imprinted chiral stationary phases has traditionally been limited by the need for a chiral pure template. Paradoxically, availability of a chiral pure template largely defeats the purpose of developing a chiral stationary phase. To solve this paradox, imprinting of scalemic and racemic template mixtures was investigated using both chiral $(\mathrm{N}-\alpha-$ bismethacryloyl-L-alanine) and achiral (N,O-bisacrylamide ethanolamine) crosslinkers. Imprinting of scalemic mixtures provided polymers capable of partial separation of Boc-tyrosine enantiomers with virtually the same results when using either the chiral or achiral crosslinker. However, the chiral crosslinker was required for chiral differentiation by the racemic imprinted polymers which were evaluated in both batch rebinding and chromatographic modes. Batch rebinding analysis revealed intersecting binding isotherms for the L- and D-Boc-tyrosine, indicating bias for the $\mathrm{D}$ or $\mathrm{L}$ enantiomer is concentration dependent. Partial chromatographic separation was achieved by the racemic imprinted polymers providing variable D or L bias in equal probability over multiple replicates of polymer synthesis. Correlation of enantiomer bias with the batch rebinding results and optimization of HPLC parameters are discussed.
\end{abstract}




\begin{abstract}
Keywords: Molecular Imprinting, chiral crosslinker, chiral selectivity, enantiomeric excess, scalemic/racemic templates, chiral stationary phase
\end{abstract}

\title{
1. Introduction
}

Nearly all examples of chiral selectivity by molecularly imprinted polymers require the use of a chiral pure template for the imprinting process.[1-5] However, there are relatively few enantiopure compounds available compared to the universe of chiral compounds which are often found (e.g. natural products) or synthesized (e.g. pharmaceuticals) in racemic or scalemic mixtures.[6] Therefore, it is of tremendous interest to broaden the capabilities of molecular imprinting beyond requiring pure enantiomers as templates, to the use of imprinting racemic or scalemic mixtures. The molecular imprinting process using a scalemic mixture (a mixture of enantiomers at a ratio other than 1:1) and similarly for a racemic mixture is outlined in Scheme 1 where monomers and scalemic template are equilibrated in solution (a), then polymerized to give the imprinted material (b), subsequently washed to leave binding sites (c) with chiral selectivity for one of the template enantiomers (d).[7]

\section{Please put Scheme 1 here}

There have been three reports of racemic imprinting that make use of a chiral functional monomer that have shown potential for racemic imprinting;[8-10] however there have not yet been any reports investigating polymers imprinting scalemic templates. Therefore based on 
previous development of chiral crosslinkers in our group,[10] a study on the use of scalemic and racemic mixtures for imprinting was investigated using the achiral crosslinker N,Obisacrylamide ethanolamine (NOBE) or the chiral crosslinker $\mathrm{N}$ - $\alpha$-bismethacryloyl-L-alanine (LNALA) in an OMNiMIP (one monomer molecularly imprinted polymer). OMNiMIPs, which imprint using one crosslinking monomer, have shown increased performance over traditional multi-monomer imprinted polymers toward both chiral selectivity and separation of a target compound in a mixture.[11-14]

There has always been a paradox with the traditional development of molecularly imprinted chiral stationary phases (MIP-CSP). In virtually all cases (with the exception of the three references noted above), a pure chiral template has been used for the formation of a MIPCSP. The paradox is that it is necessary to have the enantiopure target compound before the MIP-CSP can be fabricated; however, the enantiopure target may not be available and that may even be the driving force for fabricating the MIP-CSP in the first place. Most chiral compounds synthesized provide scalemic or racemic products, even if a stereoselective process is used.[6] Therefore, development of molecular imprinting methods that can use the scalemic or racemic compounds as the template would provide a solution to the traditional MIP-CSP paradox. Torres, et. al., demonstrated the use of a chiral monomer that forms diastereomeric complexes with each enantiomer of a racemic template, thereby forming binding sites in the MIP-CSP that exhibit chiral selective binding.[9] The same principles can be employed for scalemic templates that are used for forming MIP-CSPs.

In the racemic imprinting method presented by Torres, et. al., a non-crosslinking chiral carboxylate monomer was developed that is primarily limited to imprinting amine based targets, such as the bis(1-phenylethyl)amine. The group of Hosoya also synthesized a non-crosslinking 
functional monomer that could form Pirkle-type diastereomeric interactions with nitro-aromatic derivatized chiral amines.[8] A third example by our group introduced the chiral crosslinking monomer L-NALA, which is further investigated here toward scalemic and racemic imprinting which can ultimately be used either for enantiomeric separations, or for simply determining enantiomeric excess (\% ee) of scalemic mixtures.[10] A primary advantage of scalemic/racemic imprinted polymers is that the process time for polymerization-to-operation of the MIP-CSP can be as fast as 1-2 days; whereas commercial columns are not only expensive, but also require mobile phase development that may take longer.

\section{Experimental}

\section{Materials}

All chemicals were purchased from Sigma-Aldrich, with the exception of D-Boc-Tyr purchased from Chem-Impex, and used without further purification. The solvents were used as received from either Sigma or Fischer Scientific. Reactions carried out under anhydrous conditions were performed in oven dried glassware under $\mathrm{N}_{2}$ atmosphere. Synthesized compounds were purified by flash chromatography using flash silica gel $(32-63 \mu \mathrm{m})$ from Science Adsorbents Inc. ${ }^{1} \mathrm{H}$ NMR and ${ }^{13} \mathrm{C}$ NMR spectra were obtained on a Bruker DPX-250 and a Bruker AVIII-400 spectrometer for compounds dissolved in $\mathrm{CDCl}_{3}$. Empty stainless steel HPLC columns were purchased from Grace Davison Discovery Sciences.

\section{Synthesis of crosslinking monomers}


The synthesis of NOBE and L-NALA followed the published protocols.[10, 14] NOBE: ${ }^{1} \mathrm{H}$ NMR $\left(\mathrm{CDCl}_{3}, 400 \mathrm{MHz}\right) \delta \mathrm{ppm} 6.41(1 \mathrm{H}, \mathrm{br}, \mathrm{NH}), 6.06(1 \mathrm{H}, \mathrm{s}), 5.64(1 \mathrm{H}, \mathrm{s}), 5.54(1 \mathrm{H}, \mathrm{s})$, 5.27-5.25 (1H, d, J= $8 \mathrm{~Hz}), 4.25-4.22(2 \mathrm{H}, \mathrm{t}, \mathrm{J}=6 \mathrm{~Hz}), 3.58-3.54(2 \mathrm{H}, \mathrm{q}), 1.90(3 \mathrm{H}, \mathrm{s}), 1.88(3 \mathrm{H}$, s. ${ }^{13} \mathrm{C} \mathrm{NMR}\left(\mathrm{CDCl}_{3}, 100 \mathrm{MHz}\right) \delta \mathrm{ppm} 168.46,167.65,139.78,135.94,126.20,119.76,63.36$, 39.22, 18.58, 18.29. NALA: ${ }^{1} \mathrm{H}$ NMR $\left(\mathrm{CDCl}_{3}, 400 \mathrm{MHz}\right) \delta \mathrm{ppm} 6.12(\mathrm{~s}, 1 \mathrm{H}), 6.01(\mathrm{br}, 1 \mathrm{H})$, $5.99(\mathrm{~s}, 1 \mathrm{H}), 5.66(\mathrm{~s}, 1 \mathrm{H}), 5.59(\mathrm{~s}, 1 \mathrm{H})$ 4, 4.39-4.36 (m, 1H), 4.16-4.09 (m, 2H), $1.94(\mathrm{~s}, 6 \mathrm{H})$, $1.24(\mathrm{~d}, 3 \mathrm{H}, 4 \mathrm{~Hz}) .{ }^{13} \mathrm{C} \mathrm{NMR}\left(\mathrm{CDCl}_{3}, 100 \mathrm{MHz}\right) \delta \mathrm{ppm} 171.14,167.89,140.02,135.93,126.16$, $119.51,67.10,44.89,18.56,18.27,17.31$.

\section{Synthesis of imprinted polymers}

The monomer $(1.0 \mathrm{~g})$ was added to a $13 \times 100 \mathrm{~mm}$ glass tube along with solutions of Boc-Tyr (5 mol\% with respect to monomer) in $1.3 \mathrm{~mL}$ acetonitrile. The initiator AIBN (1.0 mol\% with respect to monomer) was added into the solution and purged with nitrogen for five minutes. To seal the system, the glass tube was capped, wrapped with Teflon tape, and overlayed with Parafilm. The glass tube was inserted into a photoreactor apparatus and submerged in a water bath where the temperature was maintained at $21^{\circ} \mathrm{C}$. The tube with the solution mixture was then exposed to a $450 \mathrm{~W}$ mercury arc lamp surrounded by a borosilicate jacket for 8 hours immersed in the water bath along with the polymer mixture. To remove the polymer, the glass test tube was broken with a hammer and the particle monolith removed. The resulting polymer was lightly crushed into pieces in the $1-5 \mathrm{~mm}$ size range and placed in a Soxhlet extraction apparatus charged with methanol for two days to remove the template(s). Using U.S.A. Standard Testing Sieves the polymer was further sized to $25-38 \mu \mathrm{m}$ after grinding with mortar and pestle and slurried with acetone. The sized polymer was slurry-packed into a stainless steel column 
(100 $\mathrm{mm} \times 2.1 \mathrm{~mm}$ i.d.) for analysis by HPLC (Hitachi L-7000 series equipped with L-7100 pump, L-7400 detector and L-7500 integrator) in a 99/1 acetonitrile/acetic acid mobile phase.

\section{Batch rebinding studies}

For batch rebinding the dry sized $(25-38 \mu \mathrm{m})$ polymer material $(50 \mathrm{mg})$ was placed into scintillation vials. In each scintillation vial filled with polymer, various concentrations ( $0.4 \mathrm{mM}-$ 1.6 mM) of L- or D-Boc-Tyr solutions ( $2 \mathrm{~mL}$ ) in 99/1 acetonitrile/acetic acid were added. The mixture was lightly shaken by hand every 2-3 hours for the first 8 hours, and allowed to sit overnight (16 hours) in order to reach equilibrium. An aliquot of the solution was removed and the absorbance directly measured by UV spectroscopy (Cary 50 UV-Vis spectrophotometer) at $278 \mathrm{~nm}$. The imprinted polymer material was regenerated by washing with acetonitrile until a peak at $278 \mathrm{~nm}$ no longer remained in the supernatant.

\section{Results and Discussion}

N,O-bisacrylamide ethanolamine (NOBE, Scheme 1) is a crosslinking monomer that was found to enhance the formation of selective binding sites in molecularly imprinted polymers (MIPs), and was further discovered to exhibit highest selectivity when used as the only monomer in the imprinting mixture.[14] This phenomenon categorized these materials as "one monomer molecularly imprinted polymers" or "OMNiMIPs", i.e. MIPs that perform better when only one crosslinking monomer is used rather than the typical use of a mixture of monomers and crosslinkers. Several examples of chiral stationary phase OMNiMIPs that have been imprinted with a single stereoisomer have been demonstrated to be effective for chiral discrimination of enantiomeric analytes.[10-13, 15] However, as pointed out in the introduction, there are 
potential advantages of imprinting scalemic or racemic templates, prompting investigation of OMNiMIP materials toward this goal. In addition to the achiral crosslinker NOBE, a related chiral crosslinker (N- $\alpha$-bismethacryloyl-L-alanine, L-NALA) has also shown success as an OMNiMIP. Thus, both NOBE and L-NALA were imprinted with scalemic and racemic mixtures of Boc-Tyr, and the chiral discrimination of Boc-Tyr enantiomers evaluated in HPLC mode. The magnitude of chromatographic retention on MIP stationary phases is a function of both the total number of binding sites and the affinity values (i.e. $K_{d}$ ) of the sites; this is meaningful for imprinting a scalemic mixture, which was anticipated to provide a greater number of binding sites for the major enantiomer thereby leading to greater retention.

\subsection{Polymers imprinted with scalemic templates}

NOBE was initially investigated toward synthesizing scalemic MIPs (referred to as NOBE-ScaleMIPs) using mixtures of Boc-Tyr with greater content of L-Boc-Tyr (75\% L : 25\% D, 50\% ee NOBE-ScaleMIP-L) in one case, and D-Boc-Tyr (25\% L : 75\% D, 50\% ee NOBEScaleMIP-D) in another. The polymers were packed into HPLC columns, and the chromatographic results showed partial separation for scalemic and racemic mixtures as shown in the cascade plot Figure S1 for NOBE-ScaleMIP-D and Figure S2 for NOBE-ScaleMIP-L in the supplementary data. For evaluation of chiral selectivity by the NOBE-ScaleMIPs, the effective separation factor $\left(\alpha^{\prime}\right)$ was determined as the ratio of capacity factors $\left(k^{\prime}\right)$ for each enantiomer run separately. As shown in Table 1, both NOBE-ScaleMIPs gave effective separation factors in the 1.5-1.8 range; and a significant observation is that binding is selective for the enantiomer that is in excess in the template mixture; i.e. the L-Boc-Tyr is more strongly 
bound to the $50 \%$ ee NOBE-ScaleMIP-L (entry 1), and vice versa (entry 2). The enantioselectivity seen is presumably due to production of an increased number of binding sites for the $\mathrm{L}$ enantiomer that proportionally results in longer retention of the $\mathrm{L}$ enantiomer over $\mathrm{D}$. This verifies the utility of NOBE OMNiMIPs for scalemic imprinting; however, Table 1 shows that imprinting a racemic mixture using this monomer results in complete lack of chiral discrimination (entry 3). The lack of enantioselectivity from imprinting a racemic template (referred to as a RaceMIP) is not unexpected because NOBE itself is achiral, and therefore all properties such as formation of pre-polymer complexes, the number of binding sites formed as a function of the pre-polymer complex concentration, and rebinding affinity of the template to the OMNiMIP must be equivalent for each enantiomer.

\section{Please Put Table 1 Here}

Scalemic imprinting was also investigated using the chiral crosslinker L-NALA that has the possibility of forming diastereomeric pre-polymer complexes with the $\mathrm{L}$ and $\mathrm{D}$ enantiomers of Boc-Tyr. Potentially, this could have an advantage toward biasing the affinity of the binding sites preferentially for the $\mathrm{L}$ or $\mathrm{D}$ enantiomer of the template. However, entries 1 and 2 in Table 2 for the L-NALA-ScaleMIPs give $\alpha^{\prime}$ values similar to the corresponding entries in Table 1 for L- and D-ScaleMIPs synthesized using the achiral NOBE crosslinker. This result discounts any significant influence of crosslinker chirality on the overall scalemic imprinting process. In addition, there is only a small influence on the enantioselectivity by the crosslinker chirality, 
seen by the comparable values of L-NALA OMNiMIPs made with $100 \%$ L- or D-Boc tyrosine as template (entries 3 and 4 respectively in Table 2).

NMR studies were carried out to determine whether there is a lack of the diastereomeric complexation between L-NALA and the Boc-Tyr enantiomers in the pre-polymer solution (Figure 1) which would discount any diastereomeric effects in the imprinted polymer. The shift of the NH proton of L-NALA exhibits a broad peak at $6.45 \mathrm{ppm}$; thus mixtures of L-NALA with L- and D-Boc-Tyr were compared with the spectra of pure L-NALA to determine any differences in the N-H shift. Figure 1 shows that addition of either L- or D-Boc-Tyr shifts the NH peak approximately $0.1 \mathrm{ppm}$ downfield in both cases. Because little diastereomeric bias was seen for the solution phase complexes of L-NALA with L-and D-Boc-Tyr, it is not surprising that the L-NALA-ScaleMIPs were not influenced by the chiral nature of the crosslinker.

\section{Please Put Table 2 Here}

The fact that ScaleMIPs (using NOBE or L-NALA) are capable of chiral discrimination can have an important impact on analysis of stereocontrolled organic reactions that produce scalemic products. To determine \%ee of scalemic products, synthetic chemists most frequently make use of chiral columns or spectroscopic methods, which often requires a trial - and - error

\section{Please put Figure 1 Here}


approach to finding the right column or reagents. Alternatively, the ScaleMIPs offer a novel approach where the scalemic products of a chiral reaction can be directly imprinted without prior

\section{Please Put Figure 2 Here}

knowledge of the \% ee, and the ScaleMIP developed within a day can be used to chromatographically quantify the $\%$ ee of the original scalemic mixture. This is demonstrated in Figure 2 which illustrates the chiral discrimination of racemic and scalemic mixtures of the BocTyr analyte by the L-NALA- based L-ScaleMIP from entry 1 in Table 2. The chromatogram cascade in Figure 2 shows partial separation of Boc-Tyr enantiomers as the percentage of Lenantiomer in the analyte mixture is increased. The order of elution is determined by the major enantiomer of the scalemic template; in this case, L-Boc-Tyr was the excess template and is represented by the peak with longer retention. To determine if resolution could be improved at higher temperatures, chromatograms of racemic Boc-Tyr were evaluated at $25^{\circ} \mathrm{C}, 40^{\circ} \mathrm{C}$, and $55^{\circ} \mathrm{C}$. As temperature was increased, the analytes tested eluted in a shorter amount of time as shown in Figure S3 (supplementary data); however, no improvement in resolution was seen. Overall it can be concluded that ScaleMIPs applied chromatographically at ordinary room temperature can predictably differentiate between two enantiomers based on the $\%$ ee of the original template. Although resolution is incomplete, deconvolution of peaks (e.g. using OriginLab Peak Fitting software) can provide a good estimate of the enantiomeric excess for scalemic (and racemic) analytes. Similar chromatographic results are also obtained for the DScaleMIPs made with L-NALA, shown in the supplementary data (Figure S4). 


\subsection{Evaluation by L-NALA imprinted with racemic Boc-tyrosine}

A more difficult case of imprinting mixed enantiomers is the use of a racemic template which requires a chiral monomer to create stereodifferentiated binding sites.[16] To investigate the possibility of racemic imprinting, four replicates of OMNiMIPs with the same formulation were synthesized using the L-NALA chiral crosslinker. The effective separation factors for the L-NALA-based RaceMIPs shown in Table 3 (entries 1-4) reveal bias in the retention of L or D

\section{Please Put Table 3 Here}

enantiomers in roughly equal probabilities. A priori, there does not seem to be an explanation of the switching behavior for enantiomer selectivity seen by chromatography. However, it was observed that once a L-NALA-RaceMIP demonstrated a particular bias for L- or D-Boc-Tyr, the material continued to sustain the same bias perpetually for all further testing. Before carrying out more studies on these OMNiMIP materials, an experiment was conducted to determine whether the chiral L-NALA crosslinker performed better when used as the only monomer for imprinting (i.e. the OMNiMIPs in Table 3, entries 1-4), versus incorporation as a comononer along with ethyleneglycol dimethacrylate (EGDMA) which provides an inert scaffold for holding the L-NALA-template interactions in place. As shown in Figure 3 when racemic BocTyr was imprinted using a crosslinker ratio of 25 mol\% L-NALA and 75mol\% EGDMA, an $\alpha$ ' of 1.05 was obtained, indicating a lack of enantiomer separation. 


\section{Please put Figure 3 Here}

However, as the amount of L-NALA was increased, the $\alpha^{\prime}$ value increased until the highest average $\alpha^{\prime}$ value of 1.4 was obtained when $100 \%$ L-NALA was used for imprinting, and thus the OMNiMIP formulation was used for further testing. As in the case of the L-NALA-ScaleMIP shown in Figure 2, a chromatogram cascade for L-NALA-RaceMIP1 (entry 1, bias for D-BocTyr) shows partial separation of Boc-Tyr enantiomers as the percentage of D-enantiomer in the analyte mixture is increased (Figure 4). The partial separation seen is commensurable to the two other published chromatograms by other groups showing only partial separation of enantiomers by polymers imprinted with racemic templates.[8,9] Similar to the ScaleMIP case, \% ee could be obtained via peak deconvolution of the L-NALA-RaceMIP chromatogram from any of the available commercial software programs; however, the absolute configuration of the most retained enantiomer will have to be determined by a separate experiment. For comparison to the L-NALA-RaceMIPs, a polymer was synthesized using a racemic crosslinker comprised of DNALA and L-NALA with pure L-Boc-Tyr as the template gave an $\alpha$ ' value of 2.1 (entry 6 in Table 3). While this $\alpha$ ' value is higher than the L-NALA imprinted with racemic Boc-Tyr,

\section{Please Put Figure 4 Here}


it's still lower than the $\alpha$ ' value of 3.0 for pure L-Boc-Tyr imprinted using L-NALA. These results show that imprinting using a racemic mixture of template or racemic crosslinker curbs the chiral recognition of the imprinted polymers; however, racemic templates appear to have a much more severe effect on lowering the enantioselectivity of imprinted materials.

Due to the dynamic process of chromatography under flow conditions that may cause variability,[17] batch rebinding studies were implemented to establish whether a consistent bias for one enantiomer exists under thermodynamic conditions. Batch rebinding measurements were taken after equilibrium was established between the template solution and L-NALA-RaceMIP, thereby providing thermodynamic affinity information for each analyte. Batch rebinding was carried out on both a L-NALA-RaceMIP with L bias (entry 2 in Table 3), and one with a D bias (entry 3 in Table 3) by incubation of $50 \mathrm{mg}$ L-NALA-RaceMIP in $2.0 \mathrm{~mL}$ of a single enantiomer of Boc-Tyr at different concentrations. From the batch rebinding data, isotherms plotted as the logarithmic function of bound template to the polymer $\left(\log \mathrm{C}_{\mathrm{b}}\right)$ versus the free template in solution $\left(\log \mathrm{C}_{\mathrm{f}}\right)$ are graphed in Figure 5. The graphs for both L-NALA-RaceMIPs give linear output in the log-log plots of the binding isotherms as anticipated for molecularly imprinted polymers;[18-24] however, it is important to note that for both L-NALA-RaceMIPs the relative slopes of the isotherm lines representing the $\mathrm{L}$ and $\mathrm{D}$ enantiomers are different. In Figure 5a, there are two fitted lines, one corresponding to the isotherm of L-Boc-Tyr and the other for DBoc-Tyr, that do not have the same slope indicating different binding behavior for each enantiomer. Log-log plots of binding isotherms between imprinted versus the non-imprinted (or strongly imprinted versus weakly imprinted) polymers have been regularly reported, verifying this to be a reasonable observation.[9, 18-22, 25-27] The difference in slopes is a known result for imprinted polymers arising from different binding affinity distributions for each 
enantiomer.[18, 19, 21] Because the different slopes also intersect, as shown in Figures 5a and $5 \mathrm{~b}$, it can be expected that the preference for binding one enantiomer over the other will change as a function of concentration.

The dependence on concentration seen in the batch rebinding studies was further investigated in the chromatographic mode by monitoring $\alpha$ ' values as a function of analyte concentration. For L-NALA-RaceMIP2 a clear selectivity is seen biased toward the D-Boc-Tyr enantiomer; however, this selectivity declines as the concentration is increased. This trend supports the behavior seen in Figure 5a where either saturation of high affinity sites or analyte overloading on the column cause the underlying selectivity loss. This behavior is virtually the

\section{Please Put Figure 5 Here}

same for the L-NALA-RaceMIP3 column (entry 2 in Table 3), with the selectivity biasing the LBoc-Tyr as the concentration is lowered. Flow rate was also investigated toward improving selectivity, with the concentration kept constant at $1 \mathrm{mM} \mathrm{L-}$ or D-Boc-Tyr. As the flow rate was decreased for both the L-NALA-RaceMIP2 and L-NALA-RaceMIP3 an increase in the $\alpha$ ' values

\section{Please Put Table 4 Here}

was evident as shown in entries 3-5 and 8-10, respectively, in Table 4. This is a known effect for MIPs that has been ascribed to increased residence time of substrates diffusing through the 
polymer affording greater exposure of the substrate to binding sites within the bulk of the polymer.[15]

As a last comparison to the L-NALA-RaceMIP binding results, a control stationary phase (Table 3 entry 5) was created by physically mixing equal amounts (50/50 : w/w) of pure L-BocTyr (Table 2 entry 3) imprinted polymer with pure D-Boc-Tyr (Table 2 entry 4) imprinted polymer. This physically mixed stationary phase was intended to mimic the racemic imprinting process which in both cases should provide equal numbers of L- and D-Boc-Tyr imprinted sites, and should in theory give the same results as the L-NALA-RaceMIP. However, this was not the case and batch rebinding isotherms of the physically mixed stationary phase showed parallel slopes (Figure 5c), which indicates identical binding affinity distributions for each enantiomer. The greater uptake for the " $\mathrm{D}$ " imprinted material versus the " $\mathrm{L}$ " imprinted polymer is explained by direct match to the larger capacity factor for the " $\mathrm{D}$ " imprinted polymer $\left(\mathrm{k}_{\mathrm{D}}{ }_{\mathrm{D}}=7.33\right.$ in Table 2 , entry 4) versus the "L" imprinted polymer $\left(k_{L}^{\prime}=6.22\right.$, entry 3$)$. For the chromatographic results of the column packed with the mixed stationary phase, the larger capacity factor of the "D" imprinted material also gives rise to the greater retention for D-Boc-Tyr versus L-Boc-Tyr. Thus, the physically mixed stationary phase acts in a predictable manner, combining directly the properties of the two different imprinted polymers for both batch rebinding and chromatographic results.

These results may shed light on the variability of D or L preference in racemic imprinted materials. If the batch rebinding isotherms are parallel for the D and L enantiomers (as a result of identical binding affinity distributions), the selectivity will be predictable as in the case of the physically mixed stationary phase. However, if different binding affinity distributions for D and $\mathrm{L}$ analytes lead to intersecting isotherms, this can lead to variable binding selectivity. The 
underlying cause for the different binding affinity distributions is not clear. It was first hypothesized that the variability could arise from interactions between the $\mathrm{D}$ and $\mathrm{L}$ template enantiomers in the polymerization mixture; however, ${ }^{1} \mathrm{H}$ NMR studies show that there is no complexation between these enantiomers in solution (Figure S5 in the supplementary data). Studies by Baggiani and coworkers have shown that "template clustering" can occur in MIPs during rebinding, which may cause non-stoichiometric multi-analyte rebinding that could result in the variable results seen; however, they also discuss the fact that it is difficult to design experiments to observe this phenomenon for non-covalently imprinted polymers.[27] A third more general explanation would involve independent random-fluctuation in the binding affinity distributions of each enantiomer, that for a single polymer results in a bias for the D or L enantiomer; but over an infinite number of racemic imprinted polymers, this would result in equal numbers of D-biased and L-biased polymers.

\section{Conclusion}

A comprehensive comparison of scalemic and racemic imprinted polymers was evaluated using OMNiMIPs fabricated from a chiral (L-NALA) and non-chiral (NOBE) crosslinker. To the best of our knowledge this is the first report of scalemic imprinted polymers, and an initial focus was to determine whether a chiral crosslinker would enhance selectivity by materials that were imprinted with a scalemic mixture of enantiomers. The results were nearly identical for the chiral L-NALA-based polymers and the achiral NOBE-based polymers for imprinting a scalemic (50\% ee) mixture of Boc-Tyr, which showed partial resolution of scalemic and racemic mixtures in chromatographic mode. Based on these findings, the selectivity in these materials was reasoned 
to be a result of an increased number of binding sites for the major isomer, and not by diastereomeric interactions that could exist between the chiral crosslinker and the template enantiomers. Support for this conclusion came from ${ }^{1} \mathrm{H}$ NMR studies that showed equivalent shifts of the N-H peak for L-NALA in the presence of D- and L-Boc-Tyr.

For imprinting a racemic mixture (i.e. RaceMIPs), a difference in chiral selectivity was observed when the chiral L-NALA crosslinker was used, versus polymers imprinted with the achiral NOBE crosslinker which did not show any chiral selectivity at all. When L-NALA was used in combination with EGDMA as a co-crosslinker, the enantioselectivity decreased, showing L-NALA imprinting works best as an OMNiMIP. Partial chromatographic separation was achieved by the L-NALA-RaceMIPs; however, roughly half of the imprinted polymers gave better retention for D-Boc-Tyr, while the other half consistently bound L-Boc-Tyr better. The underlying mechanism for the chromatographic bias for one enantiomer or the other for each RaceMIP is not clear, however a basic understanding of the behavior of these RaceMIPs has been linked to the binding affinity distribution properties of these materials determined by batch rebinding isotherms. Optimization of HPLC parameters revealed that slower flow rate and lower concentrations improved $\alpha^{\prime}$ values, while increasing temperature did not enhance chromatographic results over room temperature. Thus it can be concluded that the L-NALARaceMIPs have equal performance to previously reported racemic imprinted polymers; $[8,9]$ however, baseline chromatographic resolution of enantiomers remains a goal for the future development of chiral crosslinkers. Because both the RaceMIPs and the ScaleMIPs can be developed within a day, this provides a facile route toward determination of the $\%$ ee of the racemate or scalemate used for imprinting, using peak deconvolution for any overlapping peaks. 


\section{Acknowledgements}

The authors gratefully acknowledge funding from the National Science Foundation grant CBET0854105. D.S.M. acknowledges GAANN fellowship P200A120213 - 13

\section{References}

[1] M. Kaniewska, M. Trojanowicz, Chiral sensors based on molecularly imprinted polymers, Elsevier Ltd., 2012, pp. 175-194.

[2] N.M. Maier, W. Lindner, Chiral recognition applications of molecularly imprinted polymers: a critical review, Anal. Bioanal. Chem., 389 (2007) 377-397.

[3] B. Sellergren, Separation of enantiomers using molecularly imprinted polymers, Wiley-VCH Verlag GmbH \& Co. KGaA, 2007, pp. 399-431.

[4] D.A. Spivak, Enantioseparations by high-performance liquid chromatography using molecularly imprinted polymers, Methods in Molecular Biology (New York, NY, United States), 970 (2013) 209-220.

[5] M.P. Tiwari, A. Prasad, Molecularly imprinted polymer based enantioselective sensing devices: A review, Anal. Chim. Acta, 853 (2015) 1-18.

[6] E.M. Carreira, Yamamoto, H., Eds.-in-Chief, Comprehensive Chirality (Vols. 1-3, 8), Elsevier B.V., Oxford, UK,, 2012.

[7] M.J. Whitcombe, N. Kirsch, I.A. Nicholls, Molecular imprinting science and technology: a survey of the literature for the years 2004-2011, J. Mol. Recognit., 27 (2014) 297-401.

[8] K. Hosoya, Y. Shirasu, K. Kimata, N. Tanaka, Molecularly Imprinted Chiral Stationary Phases: Requirement for the Chiral Template, Anal. Chem., 70 (1998) 943-945.

[9] J.J. Torres, N. Gsponer, C.L. Ramirez, D.M.A. Vera, H.A. Montejano, C.A. Chesta, Experimental and theoretical studies on the enantioselectivity of molecularly imprinted polymers prepared with a chiral functional monomer, Journal of Chromatography A, 1266 (2012) 24-33.

[10] J. LeJeune, D.A. Spivak, Chiral effects of alkyl-substituted derivatives of N,Obismethacryloyl ethanolamine on the performance of one monomer molecularly imprinted polymers (OMNiMIPs), Anal Bioanal Chem, 389 (2007) 433-440.

[11] J. Le Jeune, D.A. Spivak, Analyte separation by OMNiMIPs imprinted with multiple templates, Biosens. Bioelectron., 25 (2009) 604-608. 

versus traditional molecularly imprinted polymers, J. Mol. Recognit., 22 (2009) 121-128. [13] D.A. Spivak, M. Sibrian-Vazquez, S. Houck, Discovery and development of OMNiMIPs: One MoNomer Molecularly Imprinted Polymers, Materials Research Society Symposium Proceedings, 1005E (2007).

[14] M. Sibrian-Vazquez, D.A. Spivak, Molecular Imprinting Made Easy, J. Am. Chem. Soc., 126 (2004) 7827-7833.

[15] R. Simon, S. Houck, D.A. Spivak, Comparison of particle size and flow rate optimization for chromatography using one-monomer molecularly imprinted polymers versus traditional noncovalent molecularly imprinted polymers, Anal. Chim. Acta, 542 (2005) 104-110.

[16] A. Ciogli, D. Kotoni, F. Gasparrini, M. Pierini, C. Villani, Chiral supramolecular selectors for enantiomer differentiation in liquid chromatography, Top Curr Chem, 340 (2013) 73-105.

[17] B. Toth, G. Horvai, Chromatography, solid-phase extraction, and capillary electrochromatography with MIPs, Top. Curr. Chem., 325 (2012) 267-306.

[18] A.M. Rampey, R.J. Umpleby, II, G.T. Rushton, J.C. Iseman, R.N. Shah, K.D. Shimizu, Characterization of the Imprint Effect and the Influence of Imprinting Conditions on Affinity, Capacity, and Heterogeneity in Molecularly Imprinted Polymers Using the Freundlich IsothermAffinity Distribution Analysis, Anal. Chem., 76 (2004) 1123-1133.

[19] G.T. Rushton, C.L. Karns, K.D. Shimizu, A critical examination of the use of the Freundlich isotherm in characterizing molecularly imprinted polymers (MIPs), Anal. Chim. Acta, 528 (2005) 107-113.

[20] P. Sajonz, M. Kele, G. Zhong, B. Sellergren, G. Guiochon, Study of the thermodynamics and mass transfer kinetics of two enantiomers on a polymeric imprinted stationary phase, Journal of Chromatography A, 810 (1998) 1-17.

[21] R.J. Umpleby, S.C. Baxter, A.M. Rampey, G.T. Rushton, Y. Chen, K.D. Shimizu, Characterization of the heterogeneous binding site affinity distributions in molecularly imprinted polymers, J. Chromatogr. B: Anal. Technol. Biomed. Life Sci., 804 (2004) 141-149.

[22] X. Wu, K. Goswami, K.D. Shimizu, Comparison of monofunctional and multifunctional monomers in phosphate binding molecularly imprinted polymers, J. Mol. Recognit., 21 (2008) 410-418.

[23] F.G. Tamayo, J.L. Casillas, A. Martin-Esteban, Evaluation of new selective molecularly imprinted polymers prepared by precipitation polymerization for the extraction of phenylurea herbicides, Journal of Chromatography A, 1069 (2005) 173-181.

[24] E. Turiel, C. Perez-Conde, A. Martin-Esteban, Assessment of the cross-reactivity and binding sites characterisation of a propazine-imprinted polymer using the Langmuir-Freundlich isotherm, Analyst (Cambridge, United Kingdom), 128 (2003) 137-141.

[25] S.M. Ng, R. Narayanaswamy, Molecularly imprinted polymers as optical sensing receptors: Correlation between analytical signals and binding isotherms, Anal. Chim. Acta, 703 (2011) 226233.

[26] J.J. Torres, H.A. Montejano, C.A. Chesta, Characterization of Imprinted Microbeads Synthesized via Minisuspension Polymerization, Macromol. Mater. Eng., 297 (2012) 342-352.

[27] C. Baggiani, G. Giraudi, C. Giovannoli, C. Tozzi, L. Anfossi, Adsorption isotherms of a molecular imprinted polymer prepared in the presence of a polymerizable template. Indirect evidence of the formation of template clusters in the binding site, Anal. Chim. Acta, 504 (2004) 43-52. 


\section{Scheme Legends}

Scheme 1. Outline of the process for molecular imprinting a scalemic template mixture using LNALA or NOBE crosslinkers. (a) Pre-polymerization complex; (b) imprinted polymer; (c) templates removed from polymer; (d) selective template rebinding.

\section{Figure Legends}

Figure 1. ${ }^{1} \mathrm{H}$ NMR comparison (vertical line) of N-H shift of pure NALA (a) with solution complexes of NALA with D-Boc-Tyr (b) and NALA with L-Boc-Tyr (c). A ratio of 1:1 of monomer to template was used at 4.0M concentration of each.

Figure 2. Chromatogram cascade showing partial separation for racemic and scalemic mixtures of Boc-Tyr on L-NALA-ScaleMIP-L.

Figure 3. OMNiMIP performance using L-NALA for racemic Boc-Tyr imprinting versus MIPs made using a more traditional formulation incorporating a mixture of monomers (L-NALA and EGDMA). 
Figure 4. Cascade showing chromatographic performance of racemic/ scalemic Boc-Tyr analytes on L-NALA-RaceMIP.

Figure 5. Log-log plots of isotherms fit to the Freundlich isotherm, comparing batch rebinding of tBoc-Tyr enantiomers on L-NALA-RaceMIP2 to L-NALA-RaceMIP3 and the physically mixed material comprised of 50/50 (w/w) L-Boc-Tyr and D-Boc-Tyr imprinted polymers.

\section{Scheme 1}

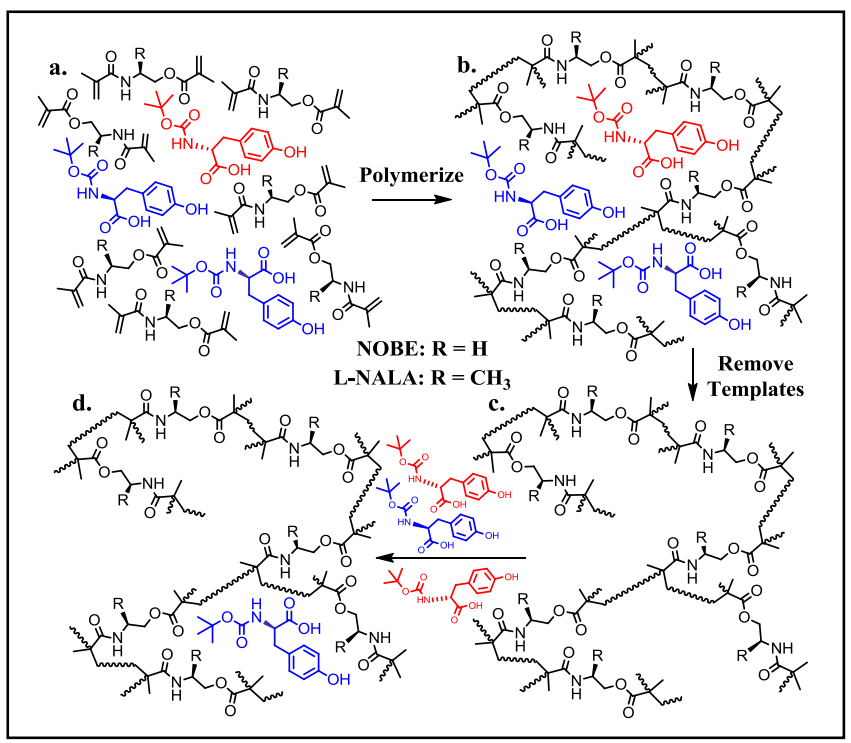


Figure 1

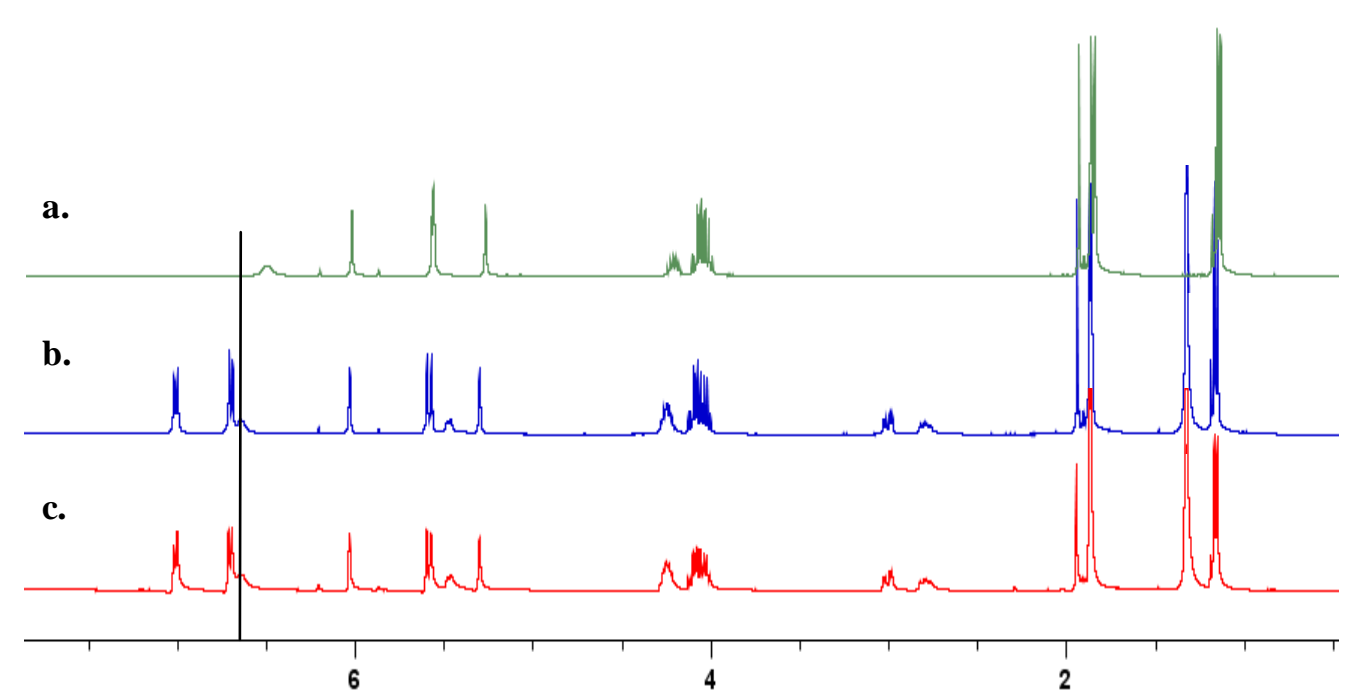

Figure 2

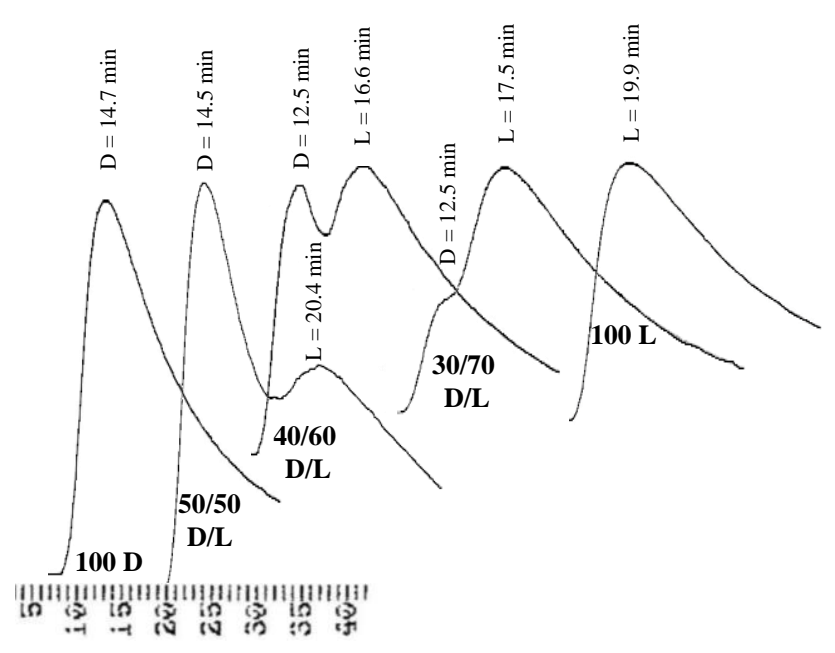




\section{Figure 3}

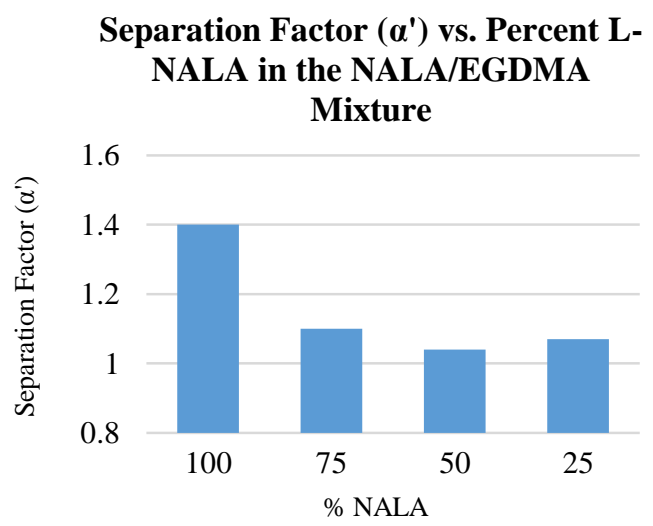

Figure 4

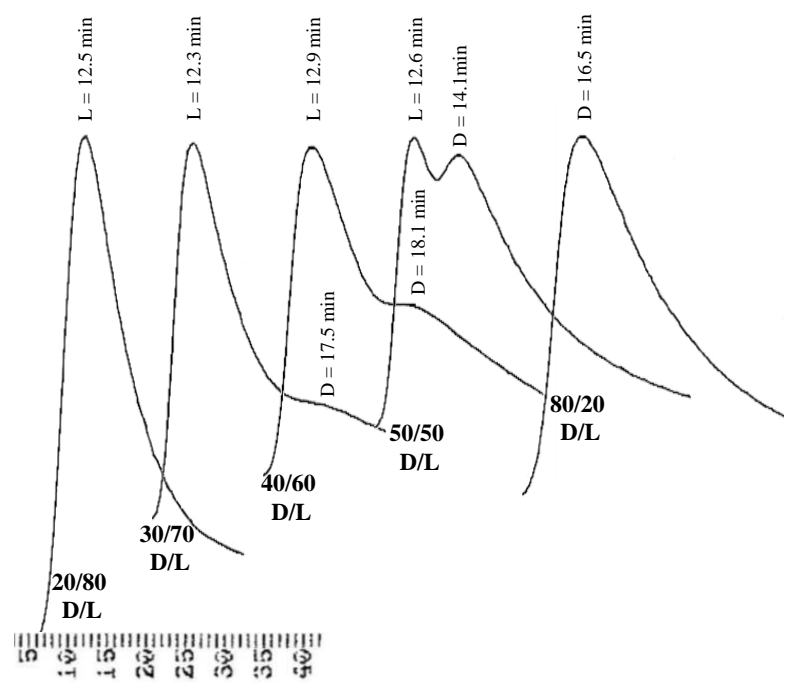




\section{Figure 5}

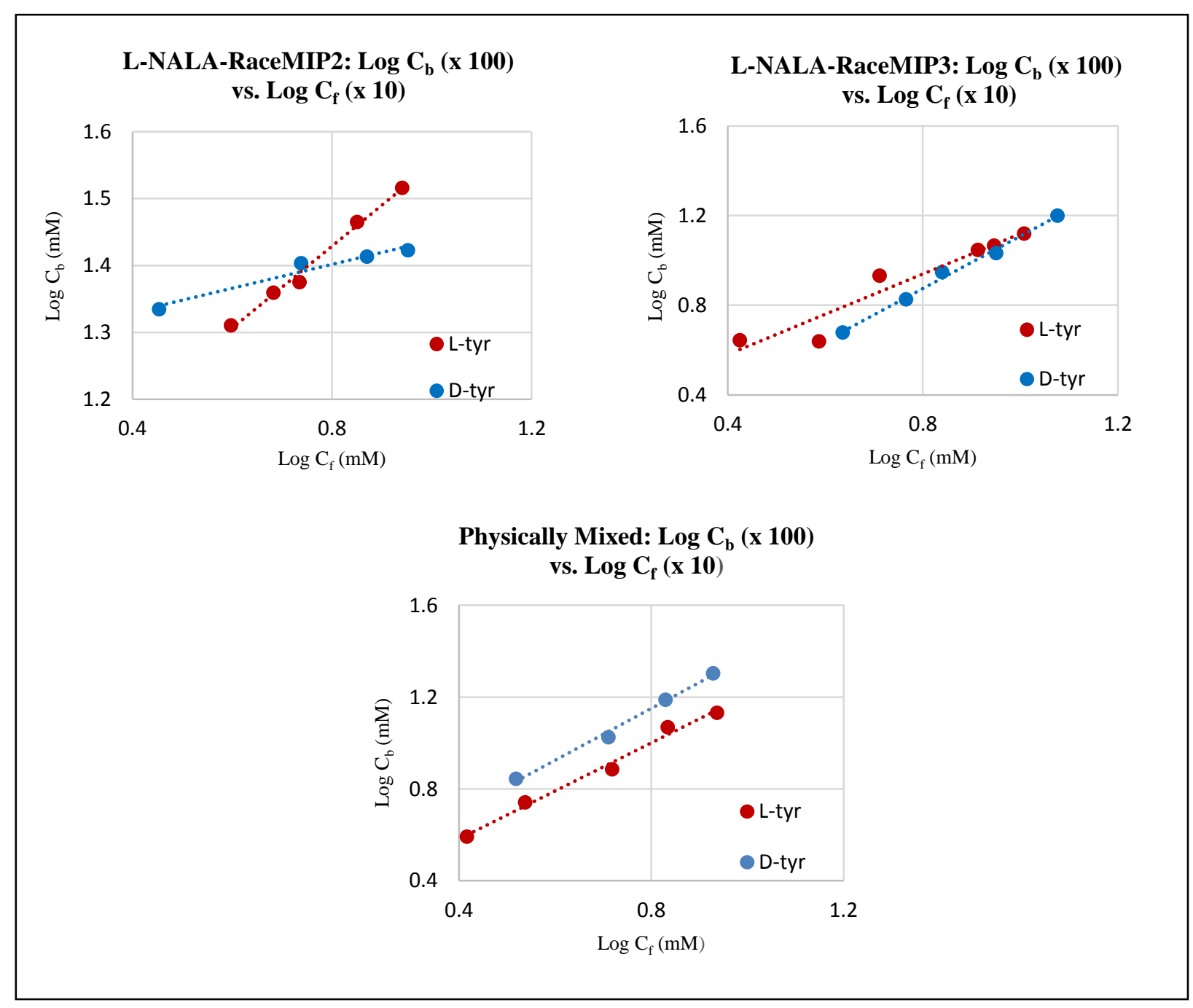




\section{Table 1}

Comparison of $\alpha^{\prime}$ values for NOBE polymers imprinted with Boc-Tyr scalemic templates versus racemic template.*

\begin{tabular}{lccccc}
\hline Polymer Identifier & Entry & Template & k' $_{\mathbf{L}}$ & $\mathbf{k}_{\mathbf{D}}^{\prime}$ & $\boldsymbol{\alpha}^{\prime}$ \\
\hline NOBE-ScaleMIP-L & 1 & $75 \% \mathrm{~L}: 25 \% \mathrm{D}$ & 5.4 & 3.5 & 1.5 \\
NOBE-ScaleMIP-D & 2 & $25 \% \mathrm{~L}: 75 \% \mathrm{D}$ & 3.1 & 5.6 & 1.8 \\
NOBE-RaceMIP & 3 & $50 \% \mathrm{~L}: 50 \% \mathrm{D}$ & 4.7 & 4.5 & 1.0
\end{tabular}

*HPLC conditions: particle size 25-37 $\mu \mathrm{m}$; column $100 \times 2.1$; mobile phase, MeCN/acetic acid (99:1); analytes (1mM Boc-L-tyrosine, $1 \mathrm{mM}$ Boc-D-tyrosine, acetone (used to determine void volume)) were all detected at $260 \mathrm{~nm}$; flow rate $0.1 \mathrm{~mL} / \mathrm{min}$; sample volume injected $5 \mu \mathrm{L}$.

Table 2

Comparison of $\alpha^{\prime}$ values for L-NALA-ScaleMIPs vs. pure L-Boc-Tyr and D-Boc-Tyr imprinted polymers.*

\begin{tabular}{cccccc}
\hline Polymer Identifier & Entry & Template & k' $_{\mathbf{L}}$ & $\mathbf{k}_{\mathbf{D}}{ }_{\mathrm{O}}$ & $\boldsymbol{\alpha}^{\prime}$ \\
\hline L-NALA-ScaleMIP-L & 1 & $75 \%$ L; 25\% D & 3.8 & 2.5 & 1.5 \\
L-NALA-ScaleMIP-D & 2 & $25 \%$ L; 75\% D & 2.1 & 3.6 & 1.7 \\
MIP-100\% L & 3 & L-Boc-Tyr & 6.2 & 2.0 & 3.1 \\
MIP-100\% D & 4 & D-Boc-Tyr & 2.3 & 7.3 & 3.0
\end{tabular}

*HPLC conditions: particle size 25-37 $\mu \mathrm{m}$; column $100 \times 2.1$; mobile phase, MeCN/acetic acid (99:1); analytes (1mM Boc-L-tyrosine, $1 \mathrm{mM}$ Boc-D-tyrosine, acetone (used to determine void volume)) were all detected at $260 \mathrm{~nm}$; flow rate $0.1 \mathrm{~mL} / \mathrm{min}$; sample volume injected $5 \mu \mathrm{L}$. 


\section{Table 3}

Comparison of $\alpha^{\prime}$ values for L-NALA-RaceMIPs vs. physically mixed and [D-NALA + LNALA] imprinted polymers.*

\begin{tabular}{|c|c|c|c|c|c|}
\hline Polymer Identifier & Entry & Template & $k_{L}^{\prime}$ & $k^{\prime}{ }_{D}$ & $\alpha^{\prime}$ \\
\hline L-NALA-RaceMIP1 & 1 & Racemic Boc-Tyr & 2.9 & 4.5 & $\begin{array}{l}1.6 \\
(\mathrm{D})^{* * *}\end{array}$ \\
\hline L-NALA-RaceMIP2 & 2 & Racemic Boc-Tyr & 3.7 & 5.1 & $\begin{array}{l}1.4 \\
(\mathrm{D})^{* * *}\end{array}$ \\
\hline L-NALA-RaceMIP3 & 3 & Racemic Boc-Tyr & 4.2 & 3.1 & $\begin{array}{l}1.4 \\
(\mathrm{~L})^{* * *}\end{array}$ \\
\hline L-NALA-RaceMIP4 & 4 & Racemic Boc-Tyr & 4.5 & 4.0 & $\begin{array}{l}1.1 \\
(\mathrm{~L}) * *\end{array}$ \\
\hline Physically Mixed & 5 & $\begin{array}{l}50 / 50(w / w) \text { L- \& D-Boc-Tyr } \\
\text { imprinted polymers }\end{array}$ & 3.6 & 4.7 & 1.3 \\
\hline $\begin{array}{c}\text { [D-NALA + L-NALA] } \\
(50 / 50: \mathrm{w} / \mathrm{w})\end{array}$ & 6 & L-Boc-Tyr & 5.1 & 2.4 & 2.1 \\
\hline
\end{tabular}

"HPLC conditions: particle size 25-37 $\mu$ m; column 100 x 2.1; mobile phase, MeCN/acetic acid (99:1); analytes (1mM Boc-L-tyrosine, $1 \mathrm{mM}$ Boc-D-tyrosine, acetone (used to determine void volume)) were all detected at $260 \mathrm{~nm}$; flow rate $0.1 \mathrm{~mL} / \mathrm{min}$; sample volume injected $5 \mu \mathrm{L}$.

** Indicates which enantiomer (D- or L-Boc-tyrosine) is retained longest. 


\section{Table 4}

Dependence of $\alpha^{\prime}$ values on analyte concentration and flow rate for L-NALA-RaceMIP2 and LNALA-RaceMIP3.*

\begin{tabular}{|c|c|c|c|c|c|c|}
\hline Polymer Identifier & Entry & $\begin{array}{c}\text { Boc-Tyr } \\
\text { concentration }\end{array}$ & $\begin{array}{l}\text { Flow Rate } \\
\text { mL/min }\end{array}$ & $\mathbf{k}_{L}^{\prime}$ & $\mathbf{K}_{\mathbf{D}}$ & $\alpha^{\prime}$ \\
\hline \multirow{3}{*}{ L-NALA-RaceMIP2 } & 1 & $5 \mathrm{mM}$ & 0.1 & 4.2 & 4.2 & 1.0 \\
\hline & 2 & $2 \mathrm{mM}$ & 0.1 & 3.7 & 4.6 & 1.2 \\
\hline & 3 & $1 \mathrm{mM}$ & 0.1 & 3.7 & 5.1 & 1.4 \\
\hline \multirow{7}{*}{ L-NALA-RaceMIP3 } & 4 & $1 \mathrm{mM}$ & 0.5 & 3.9 & 4.0 & 1.0 \\
\hline & 5 & $1 \mathrm{mM}$ & 1.0 & 2.4 & 2.6 & 1.1 \\
\hline & 6 & $5 \mathrm{mM}$ & 0.1 & 3.3 & 3.4 & 1.0 \\
\hline & 7 & $2 \mathrm{mM}$ & 0.1 & 4.0 & 3.2 & 1.3 \\
\hline & 8 & $1 \mathrm{mM}$ & 0.1 & 4.2 & 3.1 & 1.4 \\
\hline & 9 & $1 \mathrm{mM}$ & 0.5 & 3.6 & 3.6 & 1.0 \\
\hline & 10 & $1 \mathrm{mM}$ & 1.0 & 2.9 & 2.8 & 1.0 \\
\hline
\end{tabular}
(99:1); analytes (1mM Boc-L-tyrosine, $1 \mathrm{mM}$ Boc-D-tyrosine, acetone (used to determine void volume)) were all detected at $260 \mathrm{~nm}$; flow rate $0.1 \mathrm{~mL} / \mathrm{min}$; sample volume injected $5 \mu \mathrm{L}$. 